\title{
An off-board quantum point contact as a sensitive detector of cantilever motion
}

\author{
M. POGGI01,2*, M. P. JURA ${ }^{3}$, C. L. DEGEN ${ }^{1}$, M. A. TOPINKA ${ }^{4,5}$, H. J. MAMIN ${ }^{1}$, D. GOLDHABER-GORDON ${ }^{4}$ \\ AND D. RUGAR ${ }^{1}$
}

${ }^{1}$ IBM Research Division, Almaden Research Center, San Jose, California 95120, USA

${ }^{2}$ Center for Probing the Nanoscale, Stanford University, Stanford, California 94305, USA

${ }^{3}$ Department of Applied Physics, Stanford University, Stanford, California 94305, USA

${ }^{4}$ Department of Physics, Stanford University, Stanford, California 94305, USA

${ }^{5}$ Department of Material Science and Engineering, Stanford University, Stanford, California 94305, USA

*e-mail: poggio@stanford.edu

Recent advances in the fabrication of microelectromechanical systems and their evolution into nanoelectromechanical systems have enabled researchers to measure extremely small forces, masses and displacements ${ }^{1}$. In particular, researchers have developed position transducers with resolution approaching the uncertainty limit set by quantum mechanics ${ }^{2-5}$. The achievement of such resolution has implications not only for the detection of quantum behaviour in mechanical systems, but also for a variety of other precision experiments including the bounding of deviations from newtonian gravity at short distances ${ }^{6}$ and the measurement of single spins ${ }^{7}$. Here, we demonstrate the use of a quantum point contact as a sensitive displacement detector capable of sensing the low-temperature thermal motion of a nearby micromechanical cantilever. Advantages of this approach include versatility due to its off-board design, compatibility with nanoscale oscillators and, with further development, the potential to achieve quantum-limited displacement detection ${ }^{8,9}$.

At present, the most sensitive displacement detectors for nanoscale mechanical resonators rely on the mechanical modulation of current flow through a single electron transistor or atomic point contact $^{2-5}$, achieving resolutions around $10^{-15} \mathrm{~m} \mathrm{~Hz}^{-1 / 2}$, which in the case of the work by LaHaye et al. is only several times the quantum limit. These devices, however, feature a resonator and sensor integrated into a single unit, limiting their versatility for some force-sensing applications. Although high-finesse interferometers also achieve nearly quantum-limited displacement resolution-down to an astounding $10^{-20} \mathrm{~m} \mathrm{~Hz}^{-1 / 2}$ (refs 10,11) - their application to micro- and nanomechanical oscillators is challenging, especially as oscillator size is reduced. A fundamental obstacle is the optical diffraction limit, which sets a rough lower bound on the size of the measured oscillator. In addition, many requirements of a high-finesse cavity (for example, thick substrates and stiff multilayer mirror stacks for maximum reflection) run counter to the requirements of the most sensitive microelectromechanical and nanoelectromechanical systems (for example, low spring constants and thin membranes for sensitive force detection). Optical interferometers encounter some limitations at the low temperatures often required in ultrasensitive force microscopy. For temperatures below $1 \mathrm{~K}$, the absorption of light from a typical interferometer laser-even for incident powers less than $100 \mathrm{nW}$-has been observed to heat Si cantilevers
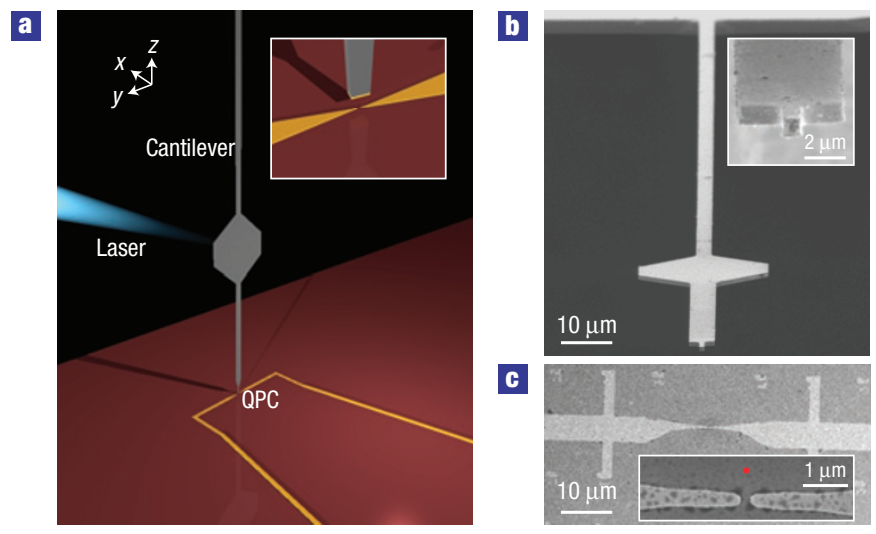

Figure 1 Components and geometry of the experiment. a, Scaled schematic diagram of the experimental set-up. A close-up view of the QPC—with the cantilever in close proximity-is shown in the inset. The laser beam is part of the low-power interferometer used to calibrate displacement measurements made by the QPC. b, Scanning electron micrograph of the cantilever and its Au-coated tip (inset). c, Scanning electron micrograph of the QPC with a high-resolution view of the active region (inset). The red dot indicates the position of the cantilever tip during the experiment.

through absorption ${ }^{12,13}$. As a result, the resolution of typical optical interferometry of micromechanical force sensors hovers above $10^{-13} \mathrm{~m} \mathrm{~Hz}^{-1 / 2}$ (refs 13,14).

Here, we show how simply bringing a micromechanical oscillator in close proximity to an off-board quantum point contact (QPC) enables sensitive displacement measurements. In demonstrating this principle, our QPC detector achieves a resolution of $10^{-12} \mathrm{~m} \mathrm{~Hz}^{-1 / 2}$, which is comparable to that achieved by optical interferometry on resonators of similar size. Our QPC transducer, however, has the fundamental advantage that it can be applied to measurements of oscillators with dimensions smaller than the optical diffraction limit. In addition, by virtue of its offboard design, the QPC can be used in conjunction with sensitive cantilevers in a variety of force-sensing applications including 


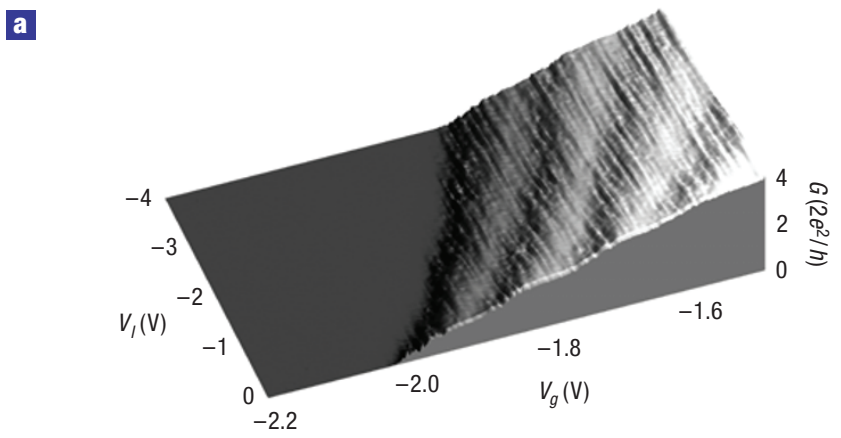

b

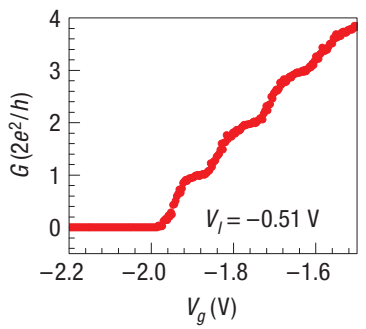

$\boldsymbol{G}$

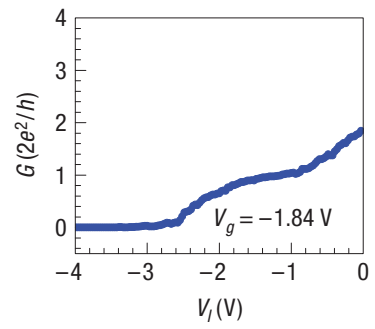

Figure 2 Pinching off the QPC using both gate voltage and lever voltage. a, Conductance plotted as a function of gate voltage and lever voltage with the cantilever tip positioned as shown in the inset of Fig. $1 \mathrm{c}$ and at $z=70 \mathrm{~nm}$ above the QPC. b,c, Linecuts of a for constant $V_{1}(\mathbf{b})$ and $V_{g}(\mathbf{c})$.

magnetic resonance force microscopy ${ }^{15}$. Although the resolution of our QPC is limited by device imperfections, QPC transducers of this type should have the properties required to reach the quantum limit on continuous position detection ${ }^{8,9}$.

In the years since the discovery of quantized conductance through semiconductor QPCs (refs 16,17), these devices have been used as sensitive charge detectors in a variety of applications. The dependence of a QPC's source-drain conductance on small changes in electrostatic fields makes it useful as a detector of single electrons in gate-defined quantum $\operatorname{dots}^{18}$ or of charge motion through electron interferometers ${ }^{19,20}$. This extreme sensitivity to charge has also been applied in the detection of mechanical motion on minute scales. In 2002, Cleland et al. demonstrated a displacement detector with a resolution of $3 \times 10^{-12} \mathrm{~m} \mathrm{~Hz}^{-1 / 2}$ at $1.5 \mathrm{MHz}$ using the piezoelectric effect in a GaAs micromechanical resonator to modulate current through an integrated QPC (ref. 21). As this method requires the QPC to be built into a piezoelectric resonator, device processing can degrade both the mobility of the two-dimensional electron gas (2DEG) forming the QPC and the quality factor of the resonator. Furthermore, the stiff doubly clamped geometry of the resonator and the requirement that it be made from a single-crystal GaAs heterostructure limit its application as a sensitive force detector. Here, we use a different scheme for displacement detection, wherein a cantilever is brought close to an off-board QPC causing the lever's motion to modulate the QPC conductance. In principle, the motion of an arbitrary resonator, without any integrated devices, can be detected in this way.

The displacement measurement, carried out in vacuum (pressure $<1 \times 10^{-6}$ torr) at $T=4.2 \mathrm{~K}$, is made by positioning the tip of a metal-coated Si cantilever about $100 \mathrm{~nm}$ above a QPC, as shown schematically in Fig. 1a. Owing to the tip's proximity to the QPC itself- the narrow channel of electron conduction directly between the gates-the lever tip and the QPC are capacitively
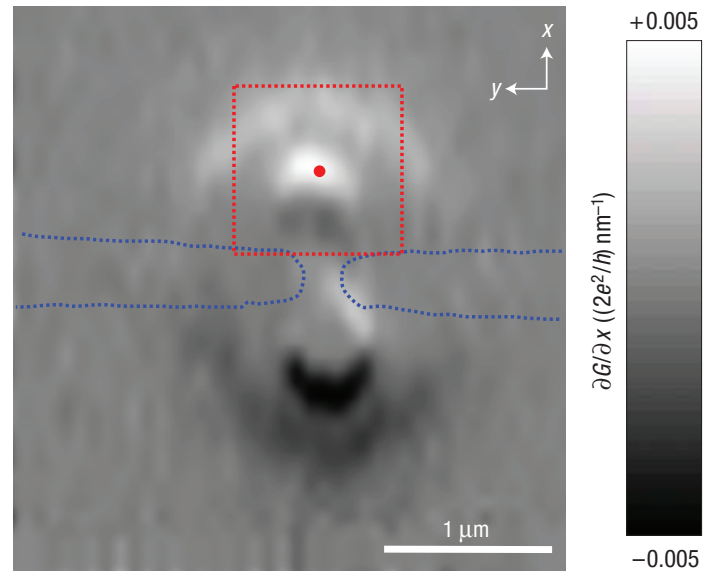

Figure $3 \partial G / \partial x$ plotted as a function of cantilever $x$ and $y$ over the QPC device. The blue dotted lines show the position of the QPC gates, whereas the red dot and red dotted lines indicate the extent of the cantilever tip and its position during the other measurements. $z=70 \mathrm{~nm}, V_{1}=-3.0 \mathrm{~V}$ and $V_{g}=-1.75 \mathrm{~V}$.

coupled. The tip acts as a movable third gate above the device surface. Changes in the cantilever potential $V_{1}$ affect the potential landscape of the QPC channel and thereby alter its conductance $G$. A voltage $V_{\mathrm{g}}$ applied to the two gates patterned on the surface modifies $G$ in the same manner.

Figure 2 shows the dependence of $G$ on both $V_{\mathrm{g}}$ and $V_{1}$ with the cantilever positioned near the QPC. The tip is located $z=70$ $\mathrm{nm}$ above the surface of the QPC device and $x=660 \mathrm{~nm}$ directly in front of the point contact, as indicated in the inset of Fig. 1c. As $V_{\mathrm{g}}$ and $V_{\mathrm{l}}$ are made more negative, both act to decrease $G$ in steps of the conductance quantum $2 e^{2} / h$ until the conductance through the point contact pinches off. From Fig. 2a,b, we determine that $G$ is about 14 times more sensitive to changes in $V_{\mathrm{g}}$ than to changes in $V_{1}$. This factor corresponds to the ratio between the gate-QPC capacitance and the tip-QPC capacitance, $C_{\mathrm{g}} / C_{\mathrm{l}}$.

The tip-QPC capacitive coupling depends strongly on their relative separation; only when the tip is positioned near the QPC does $V_{1}$ affect $G$ strongly. By moving the tip over the device surface at fixed distance $z$ and with a voltage $V_{1}$ applied, we can make an image of its effect on $G$. Because the cantilever oscillates in the $x$ direction, we are particularly interested in $\partial G / \partial x$, shown in Fig. 3. The ring pattern observed in this spatial map results from the quantized steps in conductance caused by changes in the tip-QPC separation. In regions near the point contact, where changes in the lever position most strongly affect $G$, we find a conductance response of up to $0.005\left(2 e^{2} / h\right) \mathrm{nm}^{-1}$ of cantilever motion along $x$ as shown in Fig. 3. The $x y$ position of the cantilever tip during the following experiments, also indicated in Fig. 3, corresponds to a region where $G$ responds most sensitively to changes in lever position. This response similarly depends on the $z$ position of the tip, dropping off roughly as $z^{-1}$.

With the cantilever so positioned, we study the QPC's effectiveness as a transducer of tip motion. Figure 4 shows the displacement resolution of the QPC as compared with a low-power laser interferometer simultaneously detecting the cantilever's average thermal motion. At $T=4.2 \mathrm{~K}$, the cantilever tip has a thermal motion amplitude of $x_{\mathrm{th}}=1.6 \AA_{\mathrm{r} \text {.m.s. }}$. In Fig. $4 \mathrm{a}$, the spectral density of the cantilever displacement $S_{x}$ driven by thermal force noise as measured by the optical interferometer is shown in red. The spectral density $S_{i}$ of the current driven through the point contact with a d.c. source-drain voltage $V_{\text {sd }}=2.0 \mathrm{mV}, V_{\mathrm{g}}=-1.74 \mathrm{~V}$ and 


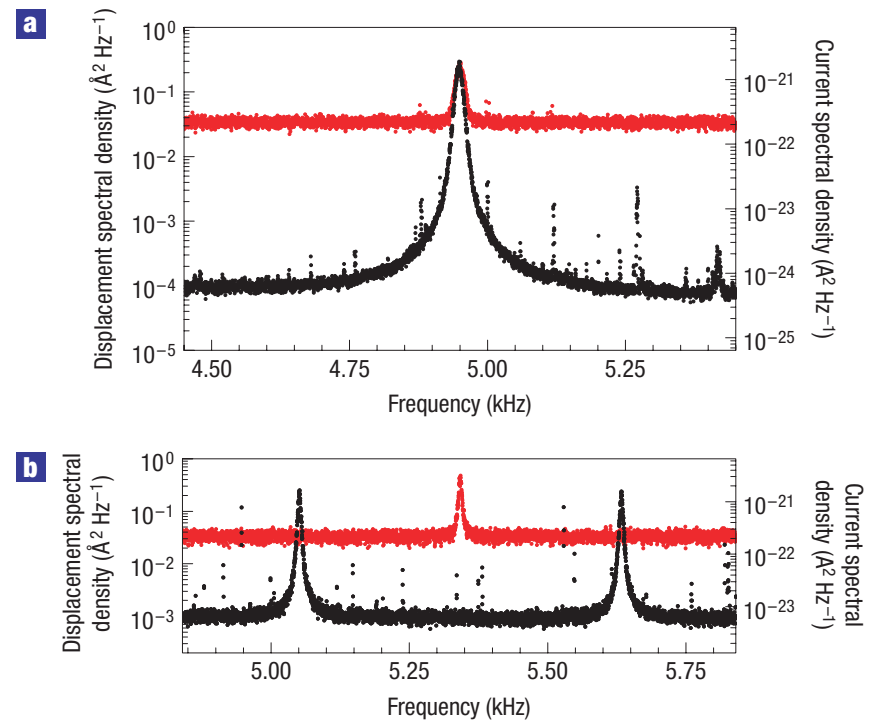

Figure 4 Cantilever thermal noise spectrum observed using a QPC transducer. a, QPC with d.c. source-drain bias. The thermal motion of the cantilever tip, measured by an optical interferometer, is plotted in red in $\AA^{2} \mathrm{~Hz}^{-1}$. The tip is $Z=70 \mathrm{~nm}$ above the QPC. The response to this motion by the QPC is plotted in black for $V_{\text {sd }}=2.0 \mathrm{mV}$ d.c., $V_{g}=-1.74 \mathrm{~V}$ and $V_{1}=-3.0 \mathrm{~V}$ in both $\mathrm{A}^{2} \mathrm{~Hz}^{-1}$ and $\AA^{2} \mathrm{~Hz}^{-1}$. b, QPC with a.c. source-drain bias. The same description applies as in $\mathbf{a}$ except that we apply a $2.0 \mathrm{mV}_{\text {r.m.s. }}$ sinusoid at $291 \mathrm{~Hz}$ to $V_{\text {sd }}$ with $V_{g}=-1.45 \mathrm{~V}$ and $V_{1}=-3.0 \mathrm{~V}$.

$V_{1}=-3.0 \mathrm{~V}$ is shown in black. The current response of the QPC matches the cantilever thermal noise in both frequency and quality factor. Once we normalize the peak QPC response to the peak amplitude of cantilever motion as measured by the interferometer, we calculate a conductance sensitivity of $0.004\left(2 e^{2} / h\right) \mathrm{nm}^{-1}$ of cantilever motion. Furthermore, this normalization enables us to plot the spectral density of the QPC response in Fig. 4, both in terms of current on the right axis and in terms of cantilever motion on the left axis. The level of the noise floor sets the resolution of the QPC displacement transducer at $10^{-12} \mathrm{~m} \mathrm{~Hz}^{-1 / 2}$, which is over an order of magnitude better than the low-power optical interferometer used here. In our experiment, the noise on the QPC current and thus the displacement resolution of the transducer is limited by charging noise (charges fluctuating near the QPC) with a $1 / f$-like dependence (see Supplementary Information, Fig. S1) ${ }^{22}$. Within the half-power bandwidth of the cantilever, this displacement noise is $4 \times 10^{-12} \mathrm{~m}$, which is over 100 times the zero-point motion of the cantilever.

To verify that the QPC response at the cantilever resonant frequency $v_{c}$ is not produced by electrical feedthrough (for example, due to stray capacitive coupling between measurement lines), we drive the QPC with an a.c. source-drain voltage $V_{\text {sd }}=2.0 \mathrm{mV}_{\text {r.m.s. }}$ at $291 \mathrm{~Hz}$. In Fig. $4 \mathrm{~b}$, the spectrum of the sourcedrain current through the QPC reveals a response centred on $\nu_{c}$ and split between two peaks spaced by twice the sourcedrain drive frequency. These sidebands are the signature of a mixer and they confirm that the response of the QPC results from the thermal motion of the nearby cantilever. The cantilever tip indeed acts as an oscillating gate that modulates the QPC conductance. Further evidence comes from exciting cantilever oscillations using a mechanically coupled piezoelectric element: the sideband amplitude increases as a function of increasing excitation amplitude.
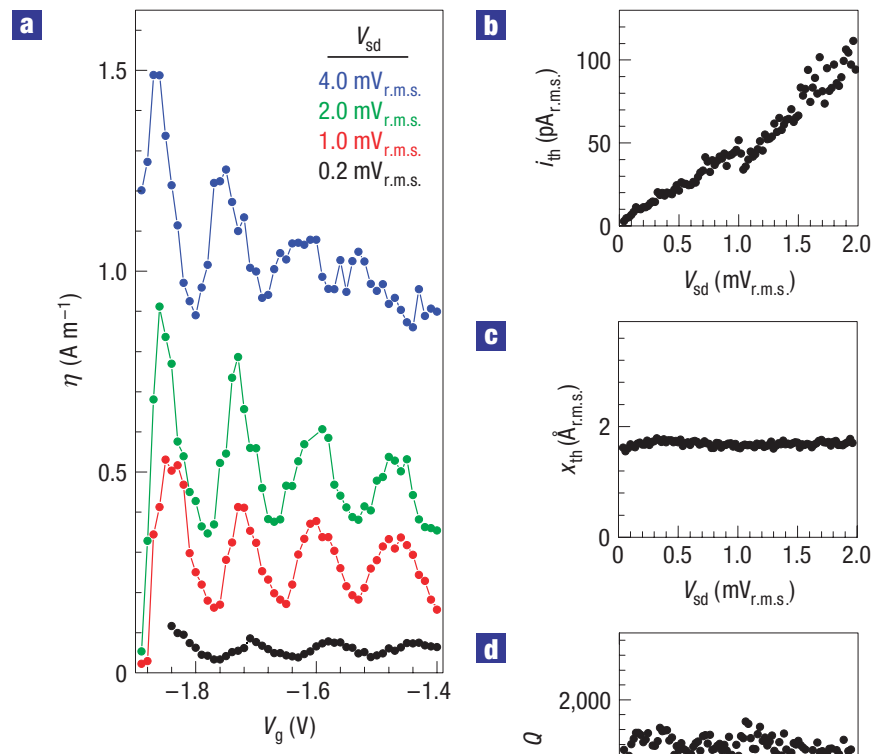

C
d
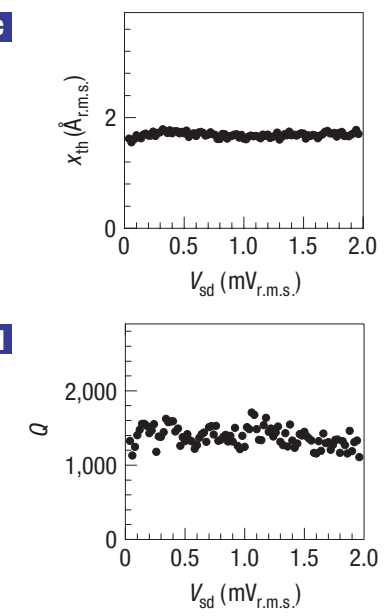

Figure 5 The response of the QPC to the thermal motion of the cantilever tip. The cantilever tip is $z=70 \mathrm{~nm}$ above the QPC with $V_{1}=-3.0 \mathrm{~V}$. a, The transduction efficiency $\eta$ plotted at different $V_{\text {sd }}$ as a function of $V_{g}$. b-d, The current response $i_{\text {th }}(\mathbf{b})$, the cantilever thermal motion $x_{\text {th }}(\mathbf{c})$ and the cantilever $Q(\mathbf{d})$ plotted as functions of $V_{\text {sd }}$ for $V_{1}=-3.0 \mathrm{~V}$ and $V_{g}=-1.8 \mathrm{~V}$.

To study the QPC's performance as a displacement transducer and to determine optimal operating conditions, we vary several parameters. Figure 5a shows the QPC transduction factor $\eta$-defined as the ratio of the cantilever's thermal motion $x_{\text {th }}$ to the QPC's corresponding current response $i_{\text {th }}$ (see the Methods section)-as a function of $V_{\mathrm{g}}$ for several values of $V_{\mathrm{sd}} \cdot \eta$ is proportional to the derivative of the conductance $G$ with respect to $V_{\mathrm{g}}$ and hence shows an oscillatory behaviour as a function of $V_{\mathrm{g}}$. As a function of $V_{\mathrm{g}}$, the maxima of $\eta$ are aligned with the steps in $G$. The steps-and indeed these oscillations in $\eta$-are manifestations of conductance quantization in QPCs. $\eta$ has a similar oscillatory dependence as a function of $V_{\mathrm{l}}$. In addition, $\eta$ increases as a function of increasing $V_{\text {sd }}$ amplitude. As shown for a fixed $V_{\mathrm{g}}$ in Fig. 5b,c, $i_{\text {th }}$ increases with increasing QPC current, whereas $x_{\text {th }}$ and $Q$ remain unaffected, even for source-drain amplitudes up to $2.0 \mathrm{mV}_{\text {r.m.s. }}$.

Whereas the cantilever $Q$ is not affected by the QPC source-drain current, it is affected by the voltages applied both to the lever and to the QPC gates. As has been observed before ${ }^{23,24}$, non-contact friction between a cantilever and a surface degrades the cantilever's $Q$ with decreasing tip-sample spacing. This degradation is exacerbated by the application of voltages to either the cantilever or the surface gates. The observed dissipation is a result of tip-sample electric fields, which can be present even when the lever and surface gates are grounded ${ }^{23}$. Under typical operating conditions $\left(V_{\mathrm{g}}=-1.75 \mathrm{~V}, V_{\mathrm{l}}=-3.0 \mathrm{~V}\right.$ and $\left.z=70 \mathrm{~nm}\right)$, the cantilever $Q$ is around 2,500, much lower than the intrinsic $Q_{0}=22,500$ measured far from the QPC surface. To minimize this external cantilever dissipation, future QPC transducers could be designed without surface gates ${ }^{25}$ and without the need to apply 
a voltage to the cantilever. Our experiments show that the QPC has a substantial response even to a grounded cantilever, possibly due to trapped charges or surface potential differences. In this case $\left(V_{\mathrm{l}}=0 \mathrm{~V}\right)$ with $V_{\text {sd }}=1 \mathrm{mV}_{\text {r.m.s. }}, V_{\mathrm{g}}=-1.98 \mathrm{~V}$ and $z=70 \mathrm{~nm}$, we find a reasonably large $\eta\left(0.16 \mathrm{~A} \mathrm{~m}^{-1}\right)$ and much larger $Q(7,500)$ than with a voltage applied to the lever.

The prospects for improving the resolution of future QPC transducers on the basis of this proof-of-principle are bright. By reducing the temperature below $4.2 \mathrm{~K}$, the conductance steps will become sharper, thus increasing the response of the QPC transducer to a given displacement. We have also observed that lower operating temperatures decrease the $1 / f$ charging noise in some QPCs. This noise currently limits our displacement resolution. Furthermore, the impact of $1 / f$ noise will diminish for smaller cantilevers with higher resonant frequencies, potentially enabling us to reach the QPC's shot-noise limit. The bandwidth of the QPC is limited to around $10^{4}$ or $10^{5} \mathrm{~Hz}$ owing to its resistance coupled with unavoidable stray cabling capacitance. Careful design of both device and material parameters to maximize the QPC transduction factor and minimize noise may also improve future results.

\section{METHODS}

In this experiment, the cantilever and QPC are mounted in a vacuum chamber at the bottom of a ${ }^{4} \mathrm{He}$ cryostat, which is isolated from environmental vibrations. A three-dimensional positioning stage with nanometre precision and stability moves the QPC relative to the cantilever. No feedback was used to stabilize the position of the cantilever tip with respect to the QPC.

The QPC device is made from a heterostructure grown by molecular-beam epitaxy on a GaAs substrate by Sumitomo Electric Industries (which no longer makes molecular-beam epitaxy samples). The structure consists of an $800 \mathrm{~nm}$ GaAs layer grown on top of the substrate, followed by a $15 \mathrm{~nm} \mathrm{Al}_{0.265} \mathrm{Ga}_{0.735} \mathrm{As}$ layer, a $40 \mathrm{~nm}$ Si-doped $\mathrm{Al}_{0.265} \mathrm{Ga}_{0.735}$ As layer and finally a $5 \mathrm{~nm}$ GaAs cap. The 2DEG lies $60 \mathrm{~nm}$ below the surface with mobility $\mu=1.0 \times 10^{6} \mathrm{~cm}^{2} \mathrm{~V}^{-1} \mathrm{~s}^{-1}$ and a carrier density $n=4.5 \times 10^{11} \mathrm{~cm}^{-2}$ at $T=4.2 \mathrm{~K}$, corresponding to a mean free path $\lambda=11 \mu \mathrm{m}$. Ti/Au $(10 / 20 \mathrm{~nm})$ gates patterned by electron-beam lithography and shown in Fig. 1c are used to define the QPC within the 2DEG. The application of a negative potential $V_{\mathrm{g}}$ between the gates and the 2DEG forms a variable-width channel through which electrons flow. The conductance $G$ of the QPC is measured between two ohmic contacts to the 2DEG on either side of the channel. A source-drain voltage $V_{\text {sd }}$ is applied across these ohmic contacts to drive the conductance.

We use a metallized cantilever made from single-crystal Si that is $350 \mu \mathrm{m}$ long, $3 \mu \mathrm{m}$ wide and $1 \mu \mathrm{m}$ thick ${ }^{24}$. At $T=4.2 \mathrm{~K}$, the cantilever has a resonant frequency $v_{\mathrm{c}}=5.2 \mathrm{kHz}$ and an intrinsic quality factor $Q_{0}=22,500$. The oscillator's spring constant is determined to be $k=2.1 \mathrm{mN} \mathrm{m}^{-1}$ through measurements of its thermal noise spectrum at several different base temperatures. The motion of the cantilever can be detected using laser light focused onto a $25-\mu \mathrm{m}$-wide paddle $100 \mu \mathrm{m}$ from its tip and reflected back into an optical fibre interferometer ${ }^{13}$. When the interferometer is in use, $20 \mathrm{nW}$ of light is incident on the lever from a temperature-tuned $1,550 \mathrm{~nm}$ distributedfeedback laser diode ${ }^{26}$. As shown in Fig. 1 b, the cantilever includes a 5 - $\mu$ m-wide section beyond the paddle, which ends with a fine $5-\mu \mathrm{m}$-long, $1-\mu \mathrm{m}$-wide tip. A thin metallic film of $\mathrm{Cr} / \mathrm{Au}(10 / 30 \mathrm{~nm})$, with $\mathrm{Cr}$ as an adhesion layer, is evaporated onto the end of the cantilever. $22 \mathrm{~nm}$ of Pt sputtered on the lever's entire surface provides a conductive path from the cantilever base to its tip. A voltage $V_{1}$ is applied to the cantilever through a pressed-indium contact at the base of the cantilever chip.

To extract parameters such as the cantilever quality factor $Q$ and average thermal motion $x_{\mathrm{th}}$, we fit the spectral density of the cantilever displacement as measured by the optical interferometer to the expected (single-sided) spectrum from a simple harmonic oscillator, $S_{x}(\omega)=\left(4 \omega_{\mathrm{c}}^{3} x_{\mathrm{th}}^{2} / Q\right)\left(1 /\left(\omega_{\mathrm{c}}^{2}-\omega^{2}\right)^{2}+\left(\omega_{\mathrm{c}} \omega / Q\right)^{2}\right)+S_{x_{n}}$, where $\omega$ is an angular frequency, $\omega_{\mathrm{c}}=2 \pi v_{\mathrm{c}}$ and $S_{x_{n}}$ is the white spectral density of the interferometer measurement noise. Similarly, we can quantify the current response $i_{\text {th }}$ of the QPC displacement transducer by fitting the spectral density of the current through the point contact to $S_{i}(\omega)=\left(4 \omega_{\mathrm{c}}^{3} i_{\text {th }}^{2} / Q\right)\left(1 /\left(\omega_{\mathrm{c}}^{2}-\omega^{2}\right)^{2}+\left(\omega_{\mathrm{c}} \omega / Q\right)^{2}\right)+S_{i_{n}}$, where $S_{i_{n}}$ is the baseline spectral density of the current measurement noise. We therefore define the QPC transduction factor as $\eta=i_{\text {th }} / x_{\text {th }}$.

$\eta$ is determined from the QPC's response to an a.c. source-drain drive voltage at $291 \mathrm{~Hz}$, as shown in Fig. 4b. We fit to this doubly peaked response rather than to the singly peaked response to a d.c. source-drain voltage to avoid spurious signals at $v_{\mathrm{c}}$. Such signals can appear because of small fluctuations $(<100 \mu \mathrm{V})$ in the virtual ground of our current amplifier. Note that the baseline noise of the displacement measurement in Fig. $4 \mathrm{~b}$ is worse than the noise in Fig. 4a because a smaller $V_{\mathrm{g}}$ is used. The measurement shown in Fig. 4a is made with all voltages chosen to optimize the QPC transducer resolution.

The dependence of $Q$ on $V_{\text {sd }}$ shown in Fig. 5d is extracted from spectra of the cantilever's thermal motion. As these spectra are averaged over several minutes, they are susceptible to slow drift in the QPC conductance due to charges rearranging in the device. The plotted $Q$ is therefore lower than the actual $Q$, which is more accurately measured to be around 2,500 using a 'ring-down' method.

\section{Received 11 March 2008; accepted 9 May 2008; published 15 June 2008.}

\section{References}

1. Schwab, K. C. \& Roukes, M. L. Putting mechanics into quantum mechanics. Phys. Today $\mathbf{5 8}$ 36-42 (2005).

2. Knobel, R. G. \& Cleland, A. N. Nanometre-scale displacement sensing using a single electron transistor. Nature 424, 291-293 (2003).

3. Naik, A. et al. Cooling a nanomechanical resonator with quantum back-action. Nature 443 , 193-196 (2006)

4. LaHaye, M. D. et al. Approaching the quantum limit of a nanomechanical resonator. Science 304, $74-77$ (2004).

5. Flowers-Jacobs, N. E., Schmidt, D. R. \& Lehnert, K. W. Intrinsic noise properties of atomic point contact displacement detectors. Phys. Rev. Lett. 98, 096804 (2007).

6. Smullin, S. J. et al. Constraints on Yukawa-type deviations from Newtonian gravity at 20 microns. Phys. Rev. D 72, 122001 (2005).

7. Rugar, D., Budakian, R., Mamin, H. J. \& Chui, B. W. Single spin detection by magnetic resonance force microscopy. Nature 430, 329-332 (2004).

8. Clerk, A. A., Girvin, S. M. \& Stone, A. D. Quantum-limited measurement and information in mesoscopic detectors. Phys. Rev. B 67, 165324 (2003).

9. Clerk, A. A. Quantum-limited position detection and amplification: A linear response perspective. Phys. Rev. B 70, 245306 (2004).

10. Caniard, T. et al. Ultrasensitive optical measurement of thermal and quantum noises. Opt. Spectrosc. 103, 225-230 (2007).

11. Arcizet, O. et al. High-sensitivity optical monitoring of a micromechanical resonator with a quantum-limited optomechanical sensor. Phys. Rev. Lett. 97, 133601 (2006).

12. Bleszynski-Jayich, A. C., Shanks, W. E. \& Harris, J. G. E. Noise thermometry and electron thermometry of a sample-on-cantilever system below 1 Kelvin. Appl. Phys. Lett. 92, 013123 (2008).

13. Mamin, H. J. \& Rugar, D. Sub-attonewton force detection at millikelvin temperatures. Appl. Phys. Lett. 79, 3358-3360 (2001).

14. Nonnenmacher, M., Vaez-Iravani, M. \& Wickramasinghe, H. K. Attractive mode force microscopy using a feedback-controlled fiber interferometer. Rev. Sci. Instrum. 63, 5373-5376 (1992).

15. Sidles, J. A. et al. Magnetic resonance force microscopy. Rev. Mod. Phys. 67, 249-265 (1995).

16. van Wees, B. J. et al. Quantized conductance of point contacts in a two-dimensional electron gas. Phys. Rev. Lett. 60, 848-850 (1988).

17. Wharam, D. A. et al. One-dimensional transport and the quantisation of the ballistic resistance. J. Phys. C 21, L209-L214 (1988).

18. Field, M. et al. Measurements of Coulomb blockade with a noninvasive voltage probe. Phys. Rev. Lett. 70, 1311-1314 (1993).

19. Buks, E. et al. Dephasing in electron interference by a 'which-path' detector. Nature 391, 871-874 (1998).

20. Sprinzak, D., Buks, E., Heiblum, M. \& Shtrikman, H. Controlled dephasing of electrons via a phase sensitive detector. Phys. Rev. Lett. 84, 5820-5823 (2000).

21. Cleland, A. N., Aldridge, J. S., Driscoll, D. C. \& Gossard, A. C. Nanomechanical displacement sensing using a quantum point contact. Appl. Phys. Lett. 81, 1699-1701 (2002).

22. Li, Y. P. et al. Low-frequency noise in transport through quantum point contacts. Appl. Phys. Lett. 57, 774-776 (1990).

23. Stipe, B. C. et al. Noncontact friction and force fluctuations between closely spaced bodies. Phys. Rev. Lett. 87, 096801 (2001).

24. Stowe, T. D. et al. Attonewton force detection using ultrathin silicon cantilevers. Appl. Phys. Lett. 71, 288-290 (1997).

25. Wesström, J.-O. J. et al. Demonstration of quantized conductance in deeply reactive ion etched $\mathrm{In}_{0.53} \mathrm{Ga}_{0.47} \mathrm{As} / \mathrm{InP}$ electron waveguides with in-plane gates. Appl. Phys. Lett. 70, 1302-1304 (1997).

26. Bruland, K. J. et al. Thermal tuning of a fiber-optic interferometer for maximum sensitivity. Rev. Sci. Instrum. 70, 3542-3544 (1999).

Supplementary Information accompanies this paper on www.nature.com/naturephysics.

\section{Acknowledgements}

We thank A. A. Clerk for useful discussions and L. S. Moore for measuring the properties of the 2DEG used here. This work was supported by the Stanford-IBM Center for Probing the Nanoscale, an NSF NSEC, grant PHY-0425897. M.P.J. acknowledges support from an NDSEG Fellowship.

\section{Author contributions}

M.P. conceived and planned the experiment in collaboration with M.P.J., C.L.D., D.G.-G. and D.R. M.P.J. prepared and characterized the QPC samples, which were fabricated by M.A.T. and M.P.J. M.P. carried out the experiment with the assistance of C.L.D., M.P.J. and H.J.M. M.P. analysed the data and wrote the paper. All authors discussed the results and contributed to the manuscript.

Author information

Reprints and permission information is available online at http://npg.nature.com/reprintsandpermissions. Correspondence and requests for materials should be addressed to M.P. 